\title{
SPACES FOR WHICH ALL COMPACT METRIC SPACES ARE REMAINDERS
}

\author{
JAMES HATZENBUHLER AND DON A. MATTSON
}

\begin{abstract}
Let $X$ be a locally compact, completely regular, Hausdorff space, and let $K(X)$ be the lattice of compactifications of $X$. Conditions on $K(X)$ and an internal condition are obtained which characterize when $X$ has all compact metric spaces as remainders.
\end{abstract}

1. Introduction. Throughout this paper $X$ will denote a noncompact, locally compact, completely regular, Hausdorff space. A remainder of $X$ is any $\alpha X-X$, where $\alpha X$ is a Hausdorff compactification of $X$. One of the major concerns in the study of remainders has been the problem of characterizing when all members of a certain class of spaces can serve as remainders for each $X$ in another class of spaces (cf. [1], [2], [3], [7], [8], [11], [12], etc.). Let $K(X)$ denote the complete lattice of compoactifications of $X$ (see [6]). The purpose of this paper is to determine those spaces for which all compact metric spaces are remainders. (Clearly, such spaces must be locally compact.) An internal characterization and a characterization in terms of $K(X)$ are obtained.

2. Characterization. In general, notation and terminology concerning remainders will follow that of [2]. For convenience, we shall say $X$ has a countable remainder whenever some $\alpha X-X$ is countably infinite. For $\alpha X, \gamma X \in K(X)$, we recall that $\alpha X>\gamma X$ if and only if there exists a continuous mapping of $\alpha X$ onto $\gamma X$ which is the identity on $X$. Let $\beta X$ denote the Stone-Cech compactification of $X$ and let $N$ denote the natural numbers.

THEOREM 2.1. For locally compact $X$, the following are equivalent:

(A) There exists a chain $\left\{\alpha_{n} X \mid n \in N\right\}$ in $K(X)$, where $\alpha_{n} X-X=\left\{a_{i}^{n} \mid i=\right.$ $\left.1, \ldots, 2^{n}\right\}$ and where $\alpha_{n+1} X>\alpha_{n} X$ under mappings $t_{n+1}$ which satisfy $t_{n+1}\left(a_{2 i-1}^{n+1}\right)=$ $t_{n+1}\left(a_{2 i}^{n+1}\right)=a_{i}^{n}$, for $i=1, \ldots, 2^{n}$.

(B) Every compact metric space is a remainder of $X$.

(C) There exists a sequence of families $\mathcal{G}_{n}$ of pairwise disjoint, nonempty, open subsets of $X$ such that for each $n \in N, \mathcal{G}_{n}=\left\{G_{i}^{n} \mid i=1, \ldots, 2^{n}\right\}$ and

(i) $G_{2 i-1}^{n+1} \cup G_{2 i}^{n+1} \subseteq G_{i}^{n}, i=1, \ldots, 2^{n}$,

(ii) $K_{n}=X-\cup\left\{G_{i}^{n} \mid i=1, \ldots, 2^{n}\right\}$ is compact,

(iii) $K_{n} \cup G_{i}^{n}$ is noncompact for each $i=1, \ldots, 2^{n}$.

Received by the editors June 19, 1980 and, in revised form, September 16, 1980.

AMS (MOS) subject classifications (1970). Primary 54D35.

Key words and phrases. Compactification, remainders, lattice of compactifications, all compact metric spaces as remainders.

(c) 1981 American Mathematical Society 0002-9939/81/0000-0334/\$01.75 
Proof. (A) implies (B). Take $\left\{\alpha_{n} X \mid n \in N\right\}$ as in (A). For each $n \in N$, let $f_{n}$ be the natural mapping of $\beta X-X$ onto $\alpha_{n} X-X$ and where $f_{n}$ is the identity on $X$ (cf. [4]). Set $U_{i}^{n}=f_{n}^{-1}\left(a_{i}^{n}\right), i=1, \ldots, 2^{n}, V_{n}=\cup\left\{U_{i}^{n} \mid 1<i<2^{n}\right.$; $i$ odd $\}$ and $W_{n}=\cup\left\{U_{i}^{n} \mid 1 \leqslant i \leqslant 2^{n} ; i\right.$ even $\}$, for each $n \in N$. Then $V_{n}$ and $W_{n}$ partition $\beta X-X$ into disjoint, nonempty open sets. Let $X_{n}=X \cup\left\{b_{n}, c_{n}\right\}$ be the two-point compactification of $X$ determined by identifying $V_{n}$ and $W_{n}$ to points $b_{n}$ and $c_{n}$, respectively. Let $p_{n}$ be the natural mapping of $\beta X$ onto $X_{n}$, for each $n \in N$. Take $Y=\times_{n \in N} X_{n}$ and denote points of $Y$ by $\left(y_{n}\right)$, where $y_{n} \in X_{n}$. Embed $X$ in $Y$ by letting $\varphi(x)=\left(y_{n}\right)$, where $y_{n}=x$, for all $n \in N$. Suppose $\left(d_{n}\right) \in Y$ satisfies $d_{n}=b_{n}$ or $d_{n}=c_{n}$, for each $n \in N$. Our aim is to show that $\mathrm{Cl}_{Y} \varphi(X)-\varphi(X)$ consists of precisely such points. To this end, let $\pi_{n}$ be the projection of $Y$ onto $X_{n}$ and suppose that $G=\pi_{n_{1}}^{-1}\left(G_{n_{1}}\right) \cap \cdots \cap \pi_{n_{k}}^{-1}\left(G_{n_{k}}\right)$ is a basic neighborhood of $\left(d_{n}\right)$ in $Y$. If $d_{n_{i}}=b_{n_{i}}$, then $p_{n_{i}}^{-1}\left(G_{n_{i}}\right)$ contains $V_{n_{i}}$ and if $d_{n_{i}}=c_{n_{n}}$, then $W_{n_{1}} \subseteq p_{n_{i}}^{-1}\left(G_{n_{i}}\right)$. Now, for $n>1, t_{n+1} \circ f_{n+1}=f_{n}$, so that for $i=1,2, \ldots, 2^{n+1}, U_{i}^{n+1} \subseteq U_{[(i+1) / 2]}^{n}$, where [ ] is the greatest integer function. It follows that some $U_{j}^{m_{k}} \subseteq p_{m_{1}}^{-1}\left(G_{m_{1}}\right)$, for all $i=$ $1, \ldots, k$. Since $U_{j}^{n_{k}}$ is nonempty and $X$ is dense in $\beta X$, we can select $w \in X$ such that $w \in p_{n_{1}}^{-1}\left(G_{n_{1}}\right) \cap \cdots \cap p_{n_{k}}^{-1}\left(G_{n_{k}}\right)$. Then $\varphi(w) \in G \cap \varphi(X)$, so that $\left(d_{n}\right) \in$ $\mathrm{Cl}_{Y} \varphi(X)-\varphi(X)$.

Next, suppose $\left(d_{n}\right) \in Y$, where each $d_{n} \in X$ but $d_{i} \neq d_{j}$, for some $i \neq j$. Then there exist disjoint neighborhoods $U$ and $V$ in $X$ of $d_{i}$ and $d_{j}$, respectively. Now $\pi_{i}^{-1}(U) \cap \pi_{j}^{-1}(V)$ is a neighborhood of $\left(d_{n}\right)$ which contains no point of $\varphi(X)$. Similarly, if, for some $i \neq j, d_{i} \in X$ and $d_{j} \in X_{j}-X$, then $\left(d_{n}\right) \notin \mathrm{Cl}_{Y} \varphi(X)-$ $\varphi(X)$. Thus, $\mathrm{CL}_{Y} \varphi(X)$ is a compactification of $X$ whose remainder is a homeomorph of the (usual) Cantor set $\mathcal{C}$. It follows from a theorem of Magill [9] that any compact metric space is a remainder of $X$.

(B) implies (C). Let $\alpha X$ be a compactification of $X$ with remainder $\mathcal{C}$. For each $n \in N$, let $\left\{A_{i}^{n} \mid i=1, \ldots, 2^{n}\right\}$ be a collection of closed subsets of $\mathcal{C}$ such that $\mathcal{C}=\cup\left\{A_{i}^{n} \mid i=1, \ldots, 2^{n}\right\}$ and $A_{2 i-1}^{n+1} \cup A_{2 i}^{n+1}=A_{i}^{n}, i=1, \ldots, 2^{n}$. In $a X$ choose disjoint open sets $H_{i}{ }^{1}$ with $A_{i}^{1} \subseteq H_{i}^{1}$, for $i=1$, 2. Set $G_{i}{ }^{1}=H_{i}^{1} \cap X$, $i=1$, 2. Clearly $K_{1}=X-\left(G_{1}{ }^{1} \cup G_{2}{ }^{1}\right)$ is compact but $K_{1} \cup G_{1}^{1}$ and $K_{1} \cup G_{2}^{1}$ are noncompact. Proceeding inductively, assume that a collection $\mathcal{G}_{n}$ has been defined as in (C). Select a pairwise disjoint family $\left\{H_{i}^{n+1} \mid i=1, \ldots, 2^{n+1}\right\}$ of open sets in $\alpha X$, such that $A_{i}^{n+1} \subseteq H_{i}^{n+1}, i=1, \ldots, 2^{n+1}$, and set $G_{2 i-1}^{n+1}=H_{2 i-1}^{n+1} \cap G_{i}^{n}$ and $G_{2 i}^{n+1}=H_{2 i}^{n+1} \cap G_{i}^{n}$. This defines $\mathcal{G}_{n+1}$ according to (C). Hence the sequence $\left\{\mathcal{G}_{n} \mid n \in N\right\}$ satisfies (C).

(C) implies (A). We utilize Magill's construction in [7] to obtain a sequence of compactifications $\left\{\alpha_{n} X \mid n \in N\right\}$ subject to (A). Accordingly, let $\alpha_{n} X-X=\left\{a_{i}^{n} \mid i\right.$ $\left.=1, \ldots, 2^{n}\right\}$, where basic neighborhoods of each $a_{i}^{n}$ are sets $\theta \cup\left\{a_{i}^{n}\right\}$, for $\theta$ open in $X$ and $\left(K_{n} \cup G_{i}^{n}\right)-\mathcal{O}$ compact.

Define mappings $t_{n+1}$ from $\alpha_{n+1} X$ onto $\alpha_{n} X$ as in (A). Evidently, each $t_{n+1}$ is continuous at points of $X$ since $X$ is locally compact. Next, let $\theta \cup\left\{a_{i}^{n}\right\}$ be any basic neighborhood of $a_{i}^{n}$. Consider $\theta \cup\left\{a_{2 i}^{n+1}\right\}$. Since $\left[\left(K_{n+1} \cup G_{2 i}^{n+1}\right)-\theta\right] \subseteq$ $\left[K_{n+1}-\mathcal{\theta}\right] \cup\left[\left(K_{n} \cup G_{i}^{n}\right)-\mathcal{\theta}\right],\left(K_{n+1} \cup G_{2 i}^{n+1}\right)-\mathcal{\theta}$ is a closed subset of a compact set. Thus $\left(K_{n+1} \cup G_{2 i}^{n+1}\right)-\mathcal{O}$ is compact and $\theta \cup\left\{a_{2 i}^{n+1}\right\}$ is a neighborhood 
of $a_{2 i}^{n+1}$ in $\alpha_{n+1} X$. It now follows that $t_{n+1}$ is continuous at $a_{2 i}^{n+1}$. In this manner $t_{n+1}$ is demonstrated to be continuous at each point of $\alpha_{n+1} X$ and the sequence $\left\{\alpha_{n} X \mid n \in N\right\}$ satisfies (A). This complete the proof.

3. Sufficiency conditions and examples. The following is immediate from Theorem 2.1.

Corollary 3.1. (A) If $X$ contains a family $\left\{G_{n} \mid n \in N\right\}$ of pairwise disjoint open sets such that $K=X-\cup\left\{G_{n} \mid n \in N\right\}$ is compact and all $K \cup G_{n}$ are noncompact, then all compact metric spaces are remainders of $X$.

(B) If $X$ is the (topological) free union of a compact space and an infinite discrete space, then all compact metric spaces are remainders of $X$.

The converse of 3.1(A) is false. For, if $X$ is the closed unit square with $C \times\{0\}$ deleted, then all compact metric spaces are remainders of $X$, but $X$ contains no family of open sets satisfying the requirements of 3.1(A).

Evidently, if $X$ satisfies (A)-(C) of Theorem 2.1, then $X$ has a countable remainder. The converse is false. If $X=W \times W^{*}$, where $W$ is the space of all countable ordinals, then $\beta X-X=W^{*}$ (cf. $8 \mathrm{~L}$ and $8 \mathrm{M}$ of [4]). Since any compact metric space which is a continuous image of $W^{*}$ must be countable or finite, it follows from Magill's theorem [9] that (B) of 2.1 cannot hold for $X$. However, $X$ has a compactification with countable remainder since $\beta X-X$ has infinitely many components (cf. [8]).

\section{REFERENCES}

1. R. E. Chandler, Continua as remainders, revisited, General Topology Appl. 8 (1978), 63-66.

2. Hausdorff compactifications, Dekker, New York, 1978.

3. R. E. Chandler and Fu-Chien Tzung, Remainders in Hausdorff compactifications, Proc. Amer. Math. Soc. 70 (1978), 196-202.

4. L. Gillman and M. Jerison, Rings of continuous functions, reprint of 1960 ed., Springer-Verlag, Berlin, 1976.

5. J. Hatzenbuhler and D. A. Mattson, A note on c-point compactifications of locally compact spaces (submitted).

6. R. G. Lubben, Concerning the decomposition and amalgamation of points, upper semi-continuous collections, and topological extensions, Trans. Amer. Math. Soc. 49 (1941), 410-466.

7. K. D. Magill, Jr., N-point compactifications, Amer. Math. Monthly 72 (1965), 1075-1081.

8. __ Countable compactifications, Canad. J. Math. 18 (1966), 616-620.

9. __ A note on compactifications, Math. Z. 94 (1966), 322-325.

10. __ More on remainders of spaces in compactifications, Bull. Acad. Polon. Sci. Ser. Sci. Math. Astronom. Phys. 18 (1970), 449-451.

11. A. Okuyama, A characterization of a space with countable infinity, Proc. Amer. Math. Soc. 28 (1971), 595-597.

12. J. W. Rogers, Jr., On compactifications with continua as remainders, Fund. Math. 70 (1971), 7-11.

13. A. K. Steiner and E. F. Steiner, Compactifications as closures of graphs, Fund. Math. 63 (1968), 221-223.

Department of Mathematics, MoOrhead State University, Moorhead, MnNesota 56560 